\title{
Characteristics of gut microbiome of obese middle-aged men in the Lublin region - preliminary report
}

\author{
Anna Gózd-Barszczewska', Maria Kozioł-Montewka 22, Piotr Barszczewski ${ }^{3}$ \\ ${ }^{1}$ Division of Cardiology with Cardiac Intensive Care Unit, Cardinal Stefan Wyszyński Hospital, Lublin, Poland \\ 2 Pope John Paul II State School of Higher Education, Biała Podlaska, Poland \\ ${ }^{3}$ Division of Gastroenterology, Cardinal Stefan Wyszyński Hospital, Lublin, Poland
}

Gózd-Barszczewska A, Kozioł-Montewka M, Barszczewski P. Characteristics of gut microbiome of obese middle-aged men in the Lublin region - preliminary report. J Pre-Clin Clin Res. 2019; 13(1): 37-41. doi: 10.26444/jpccr/105532

\begin{abstract}
Introduction and Objective. Overweight and obesity are among the main health problems in highly developed countries, including Poland. An effective fight against complications of these phenomena may be the basis for prevention and improvement of therapy of many diseases. This preliminary study is an attempt to determine the composition of the intestinal microflora in the local population of obese middle-aged men particularly exposed to the adverse consequences of overweight and obesity.

Materials and Method. Stool samples of 20 men with abdominal obesity were the material for examination. The composition of microbiome was determined using the next generation sequencing method targeting the V3-V4 hipervariable region of $16 \mathrm{~S}$ rRNA. The Greengenes gene collection was used as a reference database.

Results. This was the first attempt to determine the gut microbiome composition in a local population. The predominant components of microbiota were bacteria classified as Firmicutes (47.45\%) and Bacteroidetes (46.05\%). The most abundant Class were Clostridia (47.06\%) and Bacteroidia (46.05\%), and most abundant Order were Clostridiales (46.50\%) and Bacteroidales (45.93\%). The greatest diversity concerned the Genus category in which the most abundant groups of microorganisms were bacteria belonging to Bacteroides (26.57\%) and Prevotella (13.26\%).

Conclusions. Molecular sequencing methods allow determination of the composition of microbiome, including microorganisms that can't be identified using classical diagnostic methods. The obtained results may form the basis for further research, including larger groups of participants, aimed at determining the role of microbiota in the development of abdominal obesity and its complications. The results can also be used for comparative studies with those from other regions, and be a part of global efforts to determine human 'core microbiome'.
\end{abstract}

\section{Key words}

microbiome, obesity, abdominal obesity, gut microbiota, cardiovascular disease

\section{INTRODUCTION}

The human organism is a unique ecosystem whose functioning, apart from human genes, also affect the genes of microorganisms colonizing ecological niches within the body. All microorganisms: commensal, symbiotic and pathogenic that inhabit environment are referred to as a microbiome [1]. The development of molecular methods allowed the gaining of knowledge about microbes that were not identifiable by using classical isolation methods, as well as identification of phenotypes. During the studies on prokaryotes, sequence analysis of the highly conserved 16sRNA region was used [2]. Research conducted using this technique allowed the determination that up to $80 \%$ of the microbiome are non-cultivable organisms [3]. In 2008, the Human Microbiome Project (HMP) was initiated to develop the composition of the human microbiome. The total number of microorganisms inhabiting the human body is estimated at $10^{12}-10^{14}$, and the most numerous group are microorganisms colonizing the gastrointestinal tract [4].

Address for correspondence: Anna Gózd-Barszczewska, Stefan Cardinal Wyszyński Provincial Specialist Hospital, al. Kraśnicka 100, 20-718 Lublin, Poland

E-mail:aniagozd@o2.pl

Received: 18 November 2018; accepted: 11 March 2019; first published: 21 March 2019
The conducted studies prompted researchers from around the world to look for the role of microorganisms in the pathogenesis of some diseases. In the course of research undertaken, it was found that the intestinal microbiota of healthy people and those burdened with certain diseases, for instance: inflammatory bowel diseases, cardiovascular diseases, diabetes and obesity, as well as some cancers, such as colon cancer, differ significantly [5-9]. It should be noted that the composition of the microbiome may show significant individual differences also in the absence of diseases [4].

Data are available to document the differences in the composition of gut microbiota depending on the place of residence, which may be influenced by genetic factors, dietary habits and environmental influences [10]. In order to identify the microbiom's impact on health processes, an important role is comparing the composition of gut microbiota in different geographic regions. This may allow a global consensus to be formed regarding the composition of gut core microbiome, as well as determine the contribution of microbiota to disease processes.

Overweight and obesity are currently among the most important and the most common risk factors for cardiovascular disease in Polish society. According to data from the European Health Survey conducted in 2014, the problem of overweight and obesity concerns respectively 
$36,6 \%$ and $16,7 \%$ of Poles over 15 years of age. This problem is more common in men who are overweight or obese in $44 \%$ and $18 \%$ respectively [11].

Adipose tissue is active and its excess contributes to adverse processes affecting various organs. Unfavorable consequences of obesity contribute to occurrence of: arterial hypertension, ischemic heart disease, type 2 diabetes, kidney stone disease, polycystic ovary syndrome, sleep apnea syndrome or nonalcoholic fatty liver disease. There are also studies documenting the impact of obesity on the increased risk of different types of cancer: colon cancer, breast cancer in postmenopausal women, endometrial cancer, stomach cancer, kidney cancer, pancreatic cancer, primary liver cancer or esophageal cancer $[12,13]$. It has been documented that both overweight and obesity are unfavorable prognostic factors, and an increase in body mass index (BMI) by $5 \mathrm{~kg} / \mathrm{m}^{2}$ is associated with an increase in overall mortality by $30 \%$ [13].

\section{OBJECTIVE}

Due to the lack of the composition of microbiome in the local population, indicating the possible effect of microbiota on the occurrence of obesity, as well as the prevalence of overweight and cardiovascular diseases in Poland, this study aimed to characterize the composition of the intestinal microbiota in a population of middle-aged men with abdominal obesity. We compared our results with data obtained from other regions of the world.

\section{MATERIALS AND METHODS}

The study was conducted from 16 February 2015-5 May 2015 in the Division of Cardiology with the Cardiac Intensive Care Unit, at the Cardinal Stefan Wyszyński Hospital in Lublin, eastern Poland. The study was approved by the Bioethics Committee registered at the Lublin Medical Association (Resolution of the Bioethics Committee, No. 97/2014/KB/ VI, 16 December 2014) and all procedures performed in the study were in accordance with Helsinki Declaration and its later amendments.

Before entering the study, each participant was informed about its objectives and methods and about the possibility of resigning from participation at any time. Consent to participate in the study was obtained in writing. Each participant then received a questionnaire concerning current medical history, lifestyle and family burdens. Methods used in the study were presented by Gózd-Barszczewska et al [14]. 20 middle-aged men (45-65) who lived in the Lublin region were selected for the study. All participants met the World Health Organisation criteria of overweight or obesity; overweight was diagnosed when the body mass index (BMI) $\geq 25$, whereas obesity when the BMI was $\geq 30$ [15]. Diagnosis of abdominal obesity was made on the basis of the World Diabetes Federation guidelines and was found when the waist circumference was at least $94 \mathrm{~cm} \mathrm{[16].} \mathrm{The} \mathrm{excluding} \mathrm{criterion}$ was the intake of antimicrobials or probiotics in the period of 2 months preceding the participation in the study. Each participant was subjected to anthropometric parameters: weight, height, waist ant hip circumference.

The material collected from the participants were stool samples. Fresh faeces in the amount corresponding to the size of a walnut, or $5 \mathrm{ml}$ in the case of liquid stool, were used. Samples were taken into sterile containers. Before the next stage of the study, the strains were stored in sterile Eppendorf tubes at the temperature of $-80^{\circ} \mathrm{C}$. DNA was extracted using the QIAamp DNA Stool Mini Kit (Qiagen, Hilden, Germany). The procedure was carried out in accordance with the manufacturer's instructions. The compositions of the microbiome was determined using next generation sequencing $16 \mathrm{~S}$ rRNA method (Illumina, CA, USA). Primers targeting V3-V4 hipervariable region of $16 \mathrm{~S}$ rRNA contained additional adapters compatible with Nextera Index Kit (Illumina, CA, USA). The primers sequences were as follows: NXt_388_F: 5'- TCG TCG GCA GCG TCA GAT GTG TAT AAG AGA CAG ACW CCT ACG GGW GGC AGC AG -3' and NXt_518_R: 5'- GTC TCG TGG GCT CGG AGA TGT GTA TAA GAG ACA GAT TAC CGC GGC TGC TGG -3'.

\section{RESULTS}

In the study group, the gut microbiome was dominated by bacteria, which accounted for $99.81 \%$ of the obtained reads; Archea accounted for $0.02 \%$ reads and $0.17 \%$ were not assigned to any systematic category. 12 Phyla were identified in the study group. The microbiome was dominated by Firmicutes (47.57\%) and Bacteroidetes (46.05\%) [14] (Fig. 1).

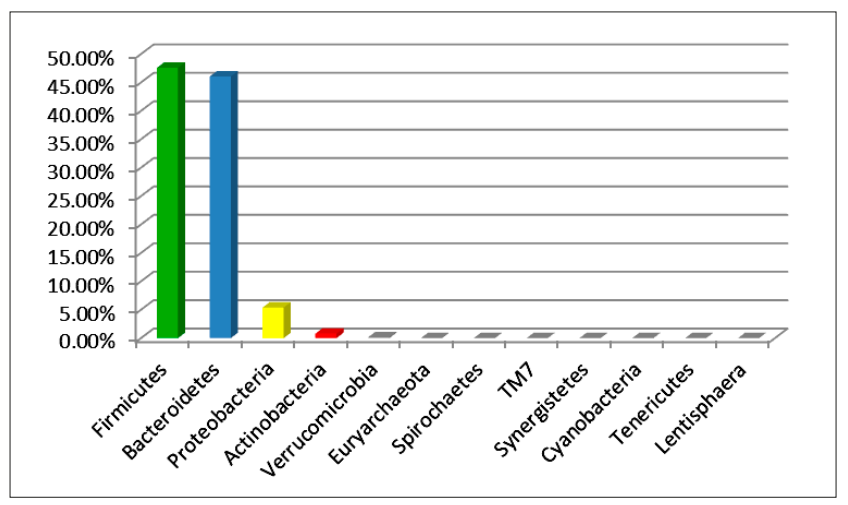

Figure 1. Phylogenetic abundance - Phylum level

In the phylogenetic category Class 20 groups of microorganisms were found. Microbiota was dominated by Clostridia (47.06\%) and Bacteroidia (46.05\%). The most abundant representatives of Proteobacteria were clusters Gammaproteobacteria and Betaproteobacteria (Fig. 2). At

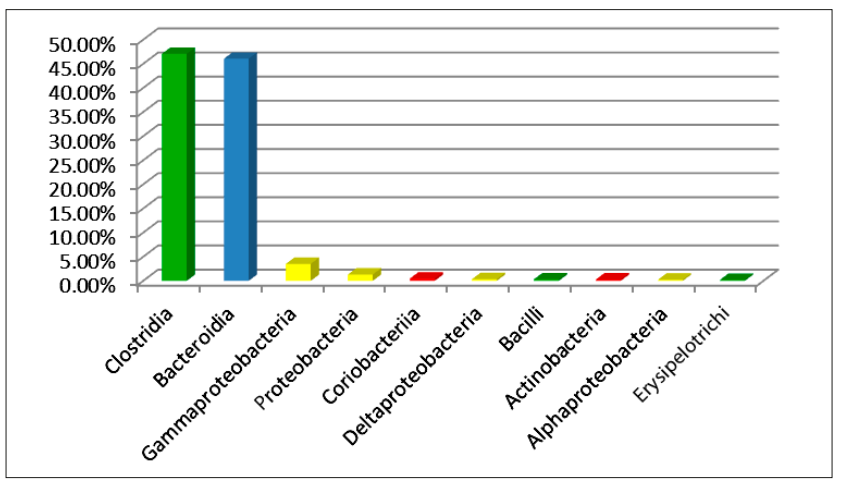

Figure 2. Phylogenetic abundance - Class level. 10 most abundant classes. Orthologous groups are colored by their respective functional category shown in Figure 1 


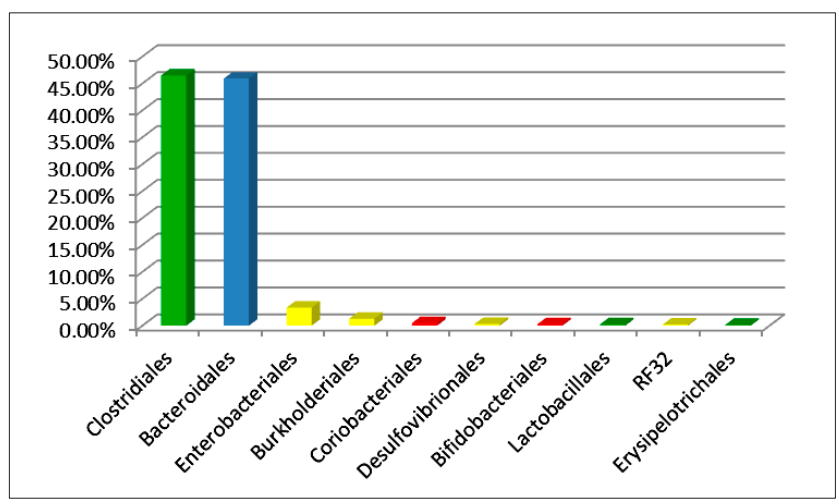

Figure 3. Phylogenetic abundance - Order level: 10 most abundant orders. Orthologous groups are coloured by their respective functional category shown in Figure 1

the Order level, 26 groups of microorganisms were identified. Most of the reads were classified as Clostridiales (46.50\%) and Bacteroidales (45.93\%) (Fig. 3).

In the study group, 45 Families were identified. The most abundant orthologous groups were Bacteroidaceae and Prevotellaceae belonging to Bacteroidetes and Ruminococcaceae, Lachnospiraceae and Veilonellaceae belonging to Firmicutes. Among the representatives of Proteobacteria, the most abundant were Enterobacteriaceae (Fig. 4) [14].

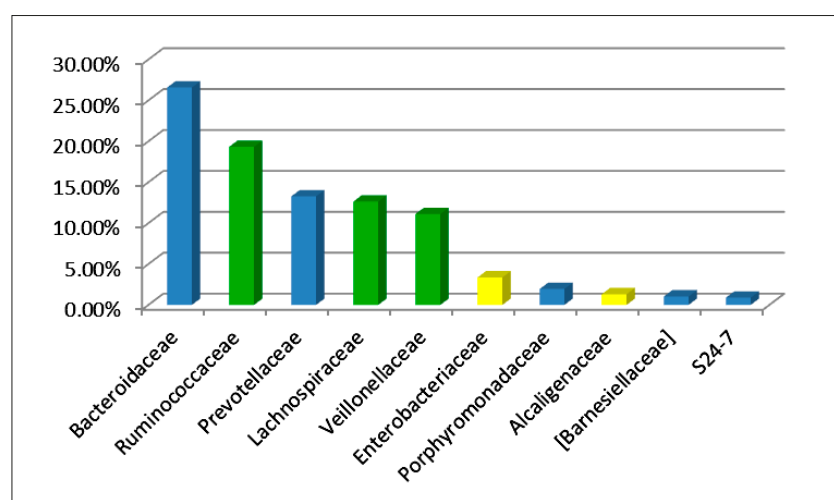

Figure 4. Phylogenetic abundance - Family level: 10 most abundant families Orthologous groups are coloured by their respective functional category shown in Figure 1

The most diverse phylogenetic category was Genus. Representatives of 67 orthologous groups were identified.

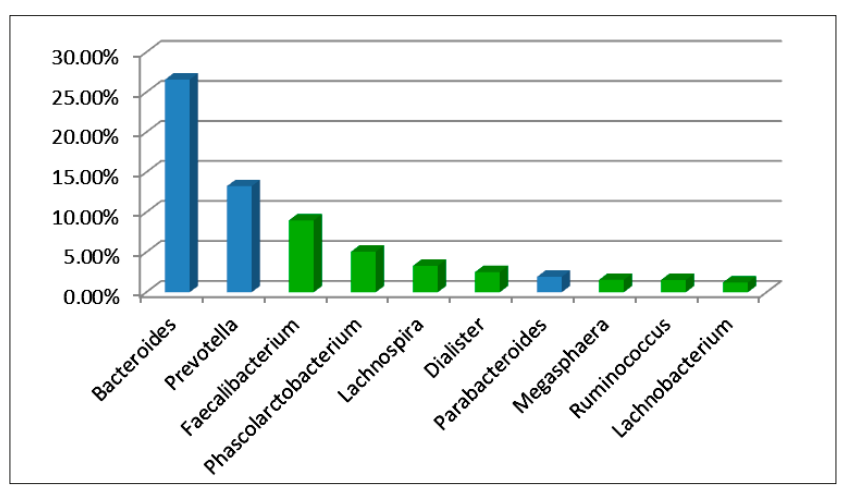

Figure 5. Phylogenetic abundance - Genus level: 10 most abundant genera Orthologous groups are coloured by their respective functional category shown in Figure 1
The dominant component of the microbiome in the study group were bacteria belonging to Bacteroides (26.57\%) and Prevotella (13.26\%) [14] (Fig. 5).

\section{DISCUSSION}

For the last few years, microbiota has been considered as a potential element of the pathogenesis of many diseases, including obesity, diabetes, and inflammatory bowel diseases. Studies conducted worldwide in recent years have confirmed the efficiency of the 16S rRNA sequencing method in the assessment of the microbiota composition and the advantage of this method over traditional techniques of culture and isolation [17]. Gut microflora begins to form at the moment of birth, and despite the influence of genetic factors, diet and environmental factors have the greatest impact on the composition of gut microbiome [18]. In adults, the microbial community appear to be stable over time [19]. The authors of the current study made the first attempt to characterize the gut microbiome in the local population of middle-aged men with abdominal obesity.

On the basis of obtained results, it was found that among 12 identified Phyla the most abundant were Firmicutes, Bacteroidetes, Proteobacteria and Actinobacteria, which constituted over $99 \%$ of gut microbiota in the study group. The most complete data obtained so far comes from the research programmes MetaHit and the Human Microbiome Project $[20,21]$. Results of these studies also revealed the presence of 12 Phyla. In the above studies, the percentage of bacteria belonging to Firmicutes, Bacteroidetes, Proteobacteria and Actinobacteria was estimated at $93.5 \%$. Similar results using the $16 \mathrm{~S}$ rRNA technique in overweight and obese middle-aged men, also with cardiovascular problems, were presented by Haro et al. [22]. The dominance of Firmicutes and Bacteroidetes can now be considered as a characteristic feature of human gut microbiome. The most numerous representatives of Firmicutes include bacteria belonging to class Clostridia, which is divided into three main families: Ruminococcaceae, Lachnospiraceae and Veillonellaceae. The most abundant class of Bacteroidetes are Bacteroidia which are represented by Bacteroidaceae and Prevotellaceae. As proved by the results of research conducted as a part of the Human Microbiome Project, microorganisms exert an unsustainable influence on the human's health through two major mechanisms: the innate immune response to the structural components of bacteria resulting in inflammation and bacterial metabolites of dietary compounds [23]. Bacteria belonging to Firmicutes and Bacteroidetes are involved in the production of short-chain fatty acids (SCAFs) which are used as energy for tissues [24]. There are numerous reports indicating the participation of bacteria in the production of SCFAs, which in turn may be involved in both processes leading to the occurrence of obesity or promote the maintenance of normal weight [25]. It has been shown that butyrate is mainly produced by the phylum Firmicutes, while acetate and propionate by Bacteroidetes [26].

The aim of current studies is to determine the impact of particular groups of microorganisms on the above processes. So far, the results of the research aimed at understanding the relationship between the composition of the intestinal microbiome and obesity still did not allow for the last determination of the role of microorganisms. There are data 
indicating significant relationships between the composition of microbiota and body weight, manifested, for example, by reduced bacterial diversity or altered ratio of Firmicutes/ Bacteroidetes [22, 27]. However, there are also studies in which no relationship was demonstrated between the composition of the gut microbiome and obesity [28].

In addition, in the studies on effect of microorganisms on the development of obesity in twins, Turnbaugh et al. have shown that $75 \%$ of the obesity-enriched genes were from Actinobacteria and 25\% from Firmicutes, while $42 \%$ of lean-enriched genes were from Bacteroidetes [27]. Although the composition of gut microbiota in the adult population is considered stable, Ley et al. have shown that the abundance of Bacteroidetes may change during weight loss [8].

The most abundant genera in the current study were Bacteroides, Prevotella, Faecalibacterium and Phascolarctobacterium. Similar results were obtained by Haro et al. in the study mentioned above. Prevotella and Bacteroides dominated especially in the gut microbiome of obese men with BMI > 33 [22]. Similar results in the composition of microbiota at the genera category were obtained in the presented study, the results which are in line with other studies conducted to-date, which showed that Bacteroides dominate in highly developed societies and constitute $40-60 \%$ of the gut microbiome [29]. Prevotella dominate in some regions of the world with a high intake of a diet based on plant-based products [30]. The abundances of Bacteroides and Prevotella in the distal colon can be inversely correlated [8].

\section{CONCLUSIONS}

Gut microbiota is an extremely complex ecosystem, which is influenced by many factors, such as genetic and environmental determinants, lifestyle and diet or medications. The aim of conducted studies is to establish the connection between microbiome composition with systemic homeostasis and its impact on human health. This was a preliminary study which was a first attempt to determine the gut microbiota composition in local population of middle-aged obese men. The results are in accordance with data obtained in other regions of the world. They can be used for comparative studies, involving larger group of participants, in order to show possible changes in the composition of gut microbiome, which may predispose to the occurrence of obesity and related diseases, and to determine whether gut microbiota may be a potential element in strategies to prevent obesity and its consequences.

\section{Conflict of interests}

Authors state that they have no conflicts of interest to disclose.

\section{REFERENCES}

1.Peterson J, Garges S, Giovanni M, McInnes P, Wang L, Schloss JA, et al. The NIH Human Microbiome Project. Genome Res. 2009; 19(12): 2317-2323. https://doi.org/10.1101/gr.096651.109.

2. Binek M. [Human microbiome - health and disease]. POST. MIKROBIOL. 2012; 51(1): 27-36 [in Polish].

3. Eckburg PB, Bik EM, Bernstein CN, Purdom E, Dethlefsen L, Sargent $\mathrm{M}$, et al. Diversity of the Human Intestinal Microbial Flora. Science. 2005; 308(5728): 1635-1638. https://doi.org/10.1126/science.1110591.
4. Wang ZK, Yang YS. Upper gastrointestinal microbiota and digestive diseases. World J Gastroenterol. 2013; 19(10): 1541-1550. https://doi. org/10.3748/wjg.v19.i10.1541

5. Frank DN, St. Amand AL, Feldman RA, Boedeker EC, Harpaz N, Pace NR. Molecular-phylogenetic characterization of microbial community imbalances in human inflammatory bowel diseases. Proc Natl Acad Sci USA. 2007; 104(34): 13780-13785. https://doi.org/10.1073/ pnas.0706625104.

6. Karlsson FH, Fåk F, Nookaew I, Tremaroli V, Fagerberg B, Petranovic $\mathrm{D}$, et al. Symptomatic atherosclerosis is associated with an altered gut metagenome. Nat Commun. 2012; 3: 1245. https://doi.org/10.1038/ ncomms 2266

7. Qin J, Li Y, Cai Z, Li S, Zhu J, Zhang F, et al. A metagenome-wide association study of gut microbiota in type 2 diabetes. Nature. 2012; 490(7418): 55-60. https://doi.org/10.1038/nature11450.

8. Ley RE, Turnbaugh PJ, Klein S, Gordon JI. Microbial ecology: Human gut microbes associated with obesity. Nature. 2006; 444(7122): 10221023.

9. Kostic AD, Chun E, Robertson L, Glickman JN, Gallini CA, Michaud M, et al. Fusobacterium nucleatum potentiates intestinal tumorigenesis and modulates the tumor immune microenvironment. Cell Host Microbe. 2013; 14(2): 207-215. https://doi.org/10.1016/j.chom.2013.07.007.

10.Lloyd-Price J, Abu-Ali G, Huttenhower C. The healthy human microbiome. Genome Med. 2016; 8: 51. https://doi.org/10.1186/s13073016-0307-y.

11. Główny Urząd Statystyczny. Stan Zdrowia ludności Polski w 2014r. [cited 26 May 2018]. Available from: http://stat.gov.pl/obszary-tematyczne/ zdrowie/zdrowie/stan-zdrowia-ludnosci-polski-w-2014-r-,6,6.html.

12. Vainio H, Kaaks R, Bianchini F. Weight control and physical activity in cancer prevention: international evaluation of the evidence. Eur J Cancer Prev. 2002; 11(Suppl 2): 94-100.

13. Jaworski P, Binda A, Tarnowski W. [The influence of obesity on the development of cancer]. Postępy nauk medycznych. 2015; 30(9): 673-676 [in Polish].

14.Gózd-Barszczewska A, Kozioł-Montewka M, Barszczewski P, Młodzińska A, Humińska K. Gut microbiome as a biomarker of cardiometabolic disorders. Ann Agric Environ Med. 2017; 24(3): $416-422$.

15. World Health Organization: Physical status: the use and interpretation of anthropometry. Report of a WHO Expert Committee. World Health Organ Tech Rep Ser. 1995; 854: 1-452. [cited 27 May 2018]. Available from: http://apps.who.int/iris/bitstream/10665/37003/1/ WHO_TRS_854.pdf.

16. Alberti G, Zimmet P, Shaw J. Metabolic syndrome - a new world - wide definition. A Consensus Statement from the International Diabetes Federation. Diabet. Med. 2006; 23: 469-480.

17. Janda JM, Abbott SL. 16S rRNA Gene Sequencing for Bacterial Identification in the Diagnostic Laboratory: Pluses, Perils, and Pitfalls. J Clin Microbiol. 200745 (9): 2761-2764. https://doi.org/10.1128/ JCM.01228-07.

18. Richards AL, Burns MB, Alazizi A, Barreiro LB, Pique-Regi R, Blekhman $\mathrm{R}$, et al. Genetic and transcriptional analysis of human host response to healthy gut microbiota. mSystems. 2016; 1(4). pii: e00067-16.

19. Faith JJ, Guruge JL, Charbonneau M, Subramanian S, Seedorf $\mathrm{H}$, Goodman AL, et al. The long-term stability of the human gut microbiota. Science. 2013; 341 (6141): 1237439. https://doi.org/10.1126/ science.1237439.

20.Li J, Jia H, Cai X, Zhong H, Feng Q, Sunagawa S, et al. An integrated catalog of reference genes in the human gut microbiome. Nat Biotechnol. 2014; 32(8): 834-841. https://doi.org/10.1038/nbt.2942.

21. Hugon P, Dufour JC, Colson P, Fournier PE, Sallah K. A comprehensive repertoire of prokaryotic species identified in human beings. Lancet Infect Dis. 2015; 15(10): 1211-1219. https://doi.org/10.1016/S14733099(15)00293-5.

22. Haro C, Rangel-Zúñiga OA, Alcalá-Díaz JF, Gómez-Delgado F, PérezMartínez P, Delgado-Lista J, et al. Intestinal Microbiota Is Influenced by Gender and Body Mass Index. PLoS One. 2016; 11(5): e0154090. https://doi.org/10.1371/journal.pone.0154090.

23. Harris K, Kassis A, Major G, Chou CJ. Is the Gut Microbiota a New Factor Contributing to Obesity and Its Metabolic Disorders? J Obes. 2012; 2012: 879151. https://doi.org/10.1155/2012/879151.

24. Rosenbaum M, Knight R, Leibel RL. The gut microbiota in human energy homeostasis and obesity. Trends Endocrinol Metab. 2015; 26(9): s. 493-501. https://doi.org/10.1016/j.tem.2015.07.002.

25. Chakraborti CK. New-found link between microbiota and obesity. World J Gastrointest Pathophysiol. 2015; 6(4): s. 110-119. https://doi. org/10.4291/wjgp.v6.i4.110. 
26. Abdallah Ismail N, Ragab SH, Abd Elbaky A, Shoeib AR, Alhosary Y, Fekry D. Frequency of Firmicutes and Bacteroidetes in gut microbiota in obese and normal weight Egyptian children and adults. Arch Med Sci. 2011; 7(3): s. 501-507. https://doi.org/10.5114/aoms.2011.23418

27. Turnbaugh PJ, Hamady M, Yatsunenko T, Cantarel BL, Duncan A, Ley RE, et al. A core gut microbiome in obese and lean twins. Nature. 2009; 457 (7228): 480-484. https://doi.org/10.1038/nature07540.

28. Finucane MM, Sharpton TJ, Laurent TJ, Pollard KS. A Taxonomic Signature of Obesity in the Microbiome? Getting to the Guts of the Matter. PLoS One. 2014; 9(1): e84689. https://doi.org10.1371/journal. pone. 0084689
29. Chiu CM, Huang WC, Weng SL, Tseng HC, Liang C, Wang WC, et al Systematic analysis of the association between gut flora and obesity through high-throughput sequencing and bioinformatics approaches. Biomed Res Int. 2014; 2014: 906168. https://doi.org/10.1155/2014/906168

30. Koren O, Knights D, Gonzalez A, Waldron L, Segata N, Knight R, et al. A Guide to Enterotypes across the Human Body: Meta-Analysis of Microbial Community Structures in Human Microbiome Datasets. PLoS Comput Biol. 2013; 9(1): e1002863. https://doi.org/10.1371/journal. pcbi.1002863. 